This is the pre-peer reviewed version of the following article:

Pérez-Tomás A., Lima A., Billon Q., Shirley I., Catalan G., Lira-Cantú M.. A Solar Transistor and Photoferroelectric Memory. Advanced Functional Materials, (2018). 28.

1707099: - . 10.1002/adfm.201707099,

which has been published in final form at https: //dx.doi.org/10.1002/adfm.201707099. This article may be used for non-commercial purposes in accordance with Wiley Terms and Conditions for Use of Self-Archived Versions. 


\title{
A solar transistor and photoferroelectric memory
}

\author{
A. Pérez-Tomás ${ }^{1 *}$, A. Lima ${ }^{1}$, Q. Billon ${ }^{1}$, I. Shirley ${ }^{1}$, G. Catalan ${ }^{* 1,2}$ and M. Lira-Cantút ${ }^{*}$ \\ ${ }^{I}$ Catalan Institute of Nanoscience and Nanotechnology (ICN2), CSIC and The Barcelona Institute of \\ Science and Technology, Campus UAB, Bellaterra, 08193 Barcelona, Spain
}

${ }^{2}$ Institut Català de Recerca i Estudis Avançats (ICREA), Barcelona 08010, Catalonia..

e-mail: *amador.perez@icn2.cat; * gustau.catalan@icn2.cat;* monica.lira@icn2.cat

Transistors switch a semiconductor conductive state (or on-state) and an insulator state (or off-state) by means of a third additional terminal known as gate [1]-[3]. An external bias between their two flow electrodes is always required for channel carriers to move, and this bias represents an undesirable yet unavoidable energy consumption. Harvesting the photon energy, a photovoltaic junction would already act as a self-powered current source, but incipient photovoltaic switches do not have a stable low energy consumption state [4][10]. Here, we report a new photo-ferroelectric transistor device concept based on a ferroelectric oxide (FEoxide)/organic semiconductor heterojunction, where an insulating FE-oxide $\left(\mathrm{Pb}\left(\mathrm{Zr}_{x} \mathrm{Ti}_{1-x}\right) \mathrm{O}_{3}\right)$ acts as a reversibly polarizable electron transport media of the organic bulk heterojunction (P3HT:PCBM) photogenerated carriers. Therefore we demonstrate two terminal (i.e. gate-less), low-cost (metal-oxide solutionprocessed), self-powered and non-volatile phototransistors that switch between bi-stable on and off states and with a low energy consumption in the off state.

A phototransistor, photo-switch or photo-FET [11]-[13] can be defined as a three-terminal device whose output can be simultaneously and independently controlled by light or voltage [14]. Ideally, the phototransistor concept should be integrated into a vertical (sandwich-like) two-terminal device for larger density of miniaturization [15] while keeping a normally-off state for better dissipation efficiency and easier control [16]. A reliable, cleanroom-less, low cost two-terminal phototransistor would represent a paradigm 
shift for the next generation of sustainable embodiments such as photodetectors, smart windows, flexible detector systems and optical memories [17]. Nevertheless, such a device is unconventional and challenging since a photovoltaic semiconductor Schottky or $p$ - $n$ junction does not switch as internal fields and chemical potentials are not bi-stable [16]. There are however several photo-switchable mechanisms that can result in diverse types of photovoltaic responses; these include ferroelectric photovoltaics [4]-[6], light-induced ionic drift in halide perovskites [7], [8], photovoltaic resistive switching [9] and the photochromic effect [10].

Among the photovoltaic mechanisms enabling switching responses, ferroelectric oxides (FE-oxide) possess useful features in their switchable properties (a changeable direction of current and voltage by polarization switching) and in their anomalous photovoltaic effect that can generate photovoltages much bigger than the band-gap [18] [19]. Nevertheless, their inherently low photocurrents (in the range of $\mathrm{nA}-\mu \mathrm{A} / \mathrm{cm}^{2}$ ) have resulted in poor overall photovoltaic efficiency [19]-[20]. A way to improve the photovoltaic current of FEoxide solar cells is through the combination with organic [31]-[34] or inorganic light absorbers [21]-[27].

It has been theoretically [28] and experimentally demonstrated [29], [30] that ferroelectrics bend their electronic band structure and offsets with respect to adjacent metals and metal-oxides when switching the ferroelectric polarization. As illustrated in Fig. 1(a), it is the objective of this study to implement this unique feature to design a new class and more compact of two terminal photovoltaic transistors. Here, we demonstrate an efficient (normally-off) gate-less (two-electrode) phototransistor where the conductivity modulation is achieved by the polarization state of a FE-oxide interlayer while the efficient light harvesting channel region is a bulk organic heterojunction. The normally-off operation is a key and unreported device enabling feature so far, and the canonical bulk photovoltaic effect currents switch between positive and negative values and no in between on and off conductivity states.

In the proposed configuration, the wide bandgap FE-oxide layer acts as both the transparent electron transport material and as a switchable interface, as shown in Fig. 1 (b). Efficient light harvesters based on organic semiconductors (OS) are defined on top the FE-oxide. OS are cheap, flexible, light-weight, made with abundant materials and have low environmental impact [35]. Usefully, they are synthesized at lower 
temperatures than oxides, so they can be grown on top of the ferroelectric layer without causing damage. As an additional advantage, the organic semiconductor absorbs light in the solar visible range, thus circumventing one of the key difficulties of working with photoferroelectrics, which is their normally wide bandgap that confines their optimum performance to the UV spectrum.

An organic bulk heterojunction is made of an electron donor (e.g. P3HT polymer) and an electron acceptor (e.g. PCBM fullerene) organic compounds; the $p$-type "donor" absorbs a photon while the $n$-type "acceptor" acquires an electron (Fig. 1 (b)). An efficient heterojunction network allows the donor polymer to contact a hole transport medium (HTM) and the acceptor fullerene to contact an electron transport medium (ETM), facilitating the extraction of charge without recombination.

The transport medium may be an oxide thin-film that (i) tunes the Schottky barrier alignment and, (ii) selects the majority carriers to avoid recombination [35]. The HTM must establish an anode ohmic contact and select holes. For this purpose, we select a thin layer of $\mathrm{V}_{2} \mathrm{O}_{5}$ [36]. Meanwhile, for the ETM we chose thin-films of the archetypical FE-oxide $\mathrm{Pb}\left(\mathrm{Zr}_{x} \mathrm{Ti}_{1-x}\right) \mathrm{O}_{3}(x=0.53)$ layer, hereafter labelled as PZT, coated on the glass/FTO substrate (see methods). Although ferroelectric PZT is often treated as an insulator, it is in fact an $n$-type widebandgap semiconductor [], so it is apt as an ETM. In addition, having switchable polarization means that its interfacial band bending and thus its contact resistivity can be tuned. This adds a new degree of freedom to the functionality of the device; specifically, the non-volatile nature of the polarization means that the photo-gated transistor can also act as a memory device.

The energy band structure and band alignment of the different layers of the (unpoled) ferroelectric photovoltaic transistor is shown in Fig. 1(c), as determined by ultraviolet photoelectron spectroscopy (UPS) and ultraviolet-visible spectrophotometry (UV-Vis) (see methods and SI). Because of the wide bandgap of the transparent conductive oxide (FTO) and the FE-oxide ETM (PZT), the great majority of the photons are able to reach the organic bulk heterojunction light absorbing layer. The photo-generated electrons and holes are then selectively collected by the ETM and HTM, (PZT and $\mathrm{V}_{2} \mathrm{O}_{5}$ respectively), which also act as barriers for minority carriers. The photo-generated electrons and holes are thus extracted from the core device at their respective metallic electrodes. 
The photovoltaic performances (in particular, the photovoltaic current density) of the proposed device have been optimized by varying the PZT layer thickness and its anneal temperature, as shown in Fig. (2). The ferroelectric PZT perovskite phase was evidenced by an XRD scan for annealing temperatures higher than 600 ${ }^{\circ} \mathrm{C}$ with a preferential orientation in $\langle 110\rangle$ (see SI). The FE-oxide layer exhibited excellent ferroelectric characteristics as shown by the typical ferroelectric hysteresis polarization-voltage curve as shown in Fig. 2(a). The remnant polarization at the FE-oxide surfaces is larger than $20 \square \mathrm{C} / \mathrm{cm}^{2}$. The FE-oxide conductivity is extremely small. At room temperature, PZT is a good dielectric insulator with a conductivity of less than $10^{-}$ $12 \square^{-1} \mathrm{~cm}^{-1}$. The bandgap of the PZT lies in the ultraviolet $(\sim 3.6 \mathrm{eV})$, making the PZT layer poorly absorbing in the visible part of the spectrum (see SI).

The ferroelectric photovoltaic properties of the FTO/PZT/Ag structure were tested under AM1.5G irradiation conditions (see SI). The area of the devices was $0.18-0.20 \mathrm{~cm}^{2}$, larger than usual for vertical photoferroelectric capacitors, which are typically micro-scale [37],[38]. In optimal conditions, only a very weak photovoltaic current of $j_{s c}=(3 \pm 2) \times 10^{-3} \mu \mathrm{A} / \mathrm{cm}^{2}$ was observed for simple photo-ferroelectric capacitors, in agreement with previous state-of-the-art large-area ferroelectric devices [39]. In contrast, when OS and HTM layers (i.e., P3HT:PCBM and $\mathrm{V}_{2} \mathrm{O}_{5}$ ) are added on top of the FTO/PZT, the photovoltaic current increases up to $j_{s c}=7.07$ $\pm 0.31 \mathrm{~mA} / \mathrm{cm}^{2}$. This is an increase of more than three orders of magnitude in comparison to the bare FE-oxide structure. The enormous increase is due to the large visible-range absorption of the organic layer, which brings additional carriers to the interface with the ferroelectric.

The ferroelectric polarization introduces non-volatile transistor-effect functionality into the photovoltaic junction as shown in Fig. 3. In the following, the poling electrode is the top silver layer, while the bottom FTO is always grounded. Switching the polarization up or down changes the ETM conduction band offset and thus modulates the FE-oxide/OS contact resistance from a state of high resistance (or off) to a state of low resistance (or on) . A FE-oxide downward poling (positive bias) results in a shift of the FE-oxide valence band towards deeper energies. Accordingly, the conduction band offset ( $\square E_{c}$ ) between the fullerene PCBM lowest unoccupied molecular orbital (LUMO n-type) and the PZT conduction band decreases. The lower energy 
barrier results in a higher electron current. Conversely, a FE-oxide upward poling (negative bias) reduces the ionization potential of valence band electrons and $\square E_{c}$ increases, and the high energy barrier results in reduced photocurrent. Meanwhile, neither downward polarization nor upward polarization [50] permits hole transport because PZT is n-type. The high resistivity of the ETM barrier layer blocks residual leakage current and sustain the off-state.

The performance of one of our on large area $\left(0.2 \mathrm{~cm}^{2}\right)$ photovoltaic transistor devices is illustrated in Fig. 4 . The standard poling process for this experiment is a DC bias applied to the silver terminal. The duration of the DC pulse was few seconds. A $\pm 20 \mathrm{~V}$ DC bias pulse is enough to switch on/off the photovoltaic transistor for the thicker PZT, while the thinnest could be switched with $\pm 5 \mathrm{~V}$. A positive poling results in negative $\square E_{c}$ and no FE-oxide/OS interface barrier, enabling photocurrents of 5-10 mA/cm ${ }^{2}$. In contrast, applying a negative voltage switches the polarization upward, thus raising the blocking barrier. This results in a drop of the photocurrent, which is only $0.01 \mathrm{~mA} / \mathrm{cm}^{2}$, a three order of magnitude difference with respect to the on-state. This off state can be reversed back applying positive bias to the anode again (DC bias $+20 \mathrm{~V} / 10 \mathrm{~s}$ ). The PVSwitch behavior is consistent after subsequent poling cycles (see Fig. 4(c)). It must be also mentioned that, consistent with the band alignment explanation, the conductivity differences upon the polarization state of the FE-oxide layer are preserved in the dark, resulting in a resistive switching device [15].

The main lesson from this investigation is that ferroelectrics can be exploited not just as photovoltaic materials in their own right, but also as (switchable) charge transport layers photoelectronic devices. In this context, ferroelectric oxides add new functionalities to the structure; in the present case, a two- electrode self-powered photo-transistor device concept has been demonstrated.

The new photo-ferroelectric transistor concept is a sandwiched organic light-absorbing layer in between an electron transport layer ( $n$-type FE-oxide) and a hole transport layer ( $p$-type metal oxide). Under sunlight illumination, the absorbing organic bulk heterojunction acts a self-powered source of photo-generated electron and holes that are driven towards their respective electrodes. Conventional photodiodes do not switch, but here, the modification of the electron transport layer barrier offsets due to the ferroelectric polarization enables 
the on-off switching of the photodiode. Additionally, the conventional phototransistor's area usage and interconnection complexity is greatly enhanced by moving from the standard in-plane three-electrode architecture to a vertical, two-electrode photodiode-like architecture.

While the new device concept may be described as a photo-transistor, the present design is multifunctional and may be used for other photonic functionalities. For example, the present device is also photonic ferroelectric random-access memory (FRAM) where the bits are written via an electrical pulse, but read using a light pulse that excite a high current in the on state, but low current in the off state. 


\section{Methods}

\section{Materials}

The synthesis of the PZT was done as follows: $\mathrm{Zr}$-isopropoxide $\left(\mathrm{Zr}\left[\mathrm{OCH}\left(\mathrm{CH}_{3}\right)_{2}\right]_{4}-70\right.$ wt. \% in 1-propanolSigma) and Ti-isopropoxide (Ti[OCH- $\left.\left(\mathrm{CH}_{3}\right)_{2}\right]_{4}(97 \%)$ Sigma) were mixed and then diluted in acetic acid and n-propanol. Afterwards, $\mathrm{Pb}$ acetate (10\% excess) [lead(II) acetate trihydrate, $\mathrm{Pb}\left(\mathrm{CH}_{3} \mathrm{CO}_{2}\right)_{2} \bullet 3 \mathrm{H}_{2} \mathrm{O}(99.999 \%)$ ] was dissolved into the above solution heating the solution in a silicon oil bath to $85{ }^{\circ} \mathrm{C}$ for dissolving the $\mathrm{Pb}$ precursor completely. After the cooling down of the solution, acetic acid and distilled water were added to modify the $\mathrm{Pb}-\mathrm{Zr}$-Ti solution and adjust the solution to make $40 \mathrm{ml} 0.4 \mathrm{M}$. The active polymer:fullerene layer was formed mixing 98\% rr-poly(3-hexylthiophene-2,5-diyl) (P3HT) and PCBM ([6,6]-Phenyl-C61-butyric acid methyl ester,99.5\%). Synthesis of the $\mathrm{V}_{2} \mathrm{O}_{5} \cdot \mathrm{H}_{2} \mathrm{O}$ xerogel was carried out by the cationic exchange method. Briefly, $4.5 \mathrm{~g}$ of sodium metavanadate $\left(\mathrm{NaVO}_{3}\right.$; Sigma) were dissolved in water at $80{ }^{\circ} \mathrm{C}$. Once cold, the solution was passed through a cationic exchange DOWEX50 WX2 50-100 (Sigma). The initial colourless solution changed to yellow-orange corresponding to the formation of the metavanadic acid. The polycondensation of the vanadic acid results in the formation of the $\mathrm{V}_{2} \mathrm{O}_{5}$ gel (red) after one month standing at room temperature. The final sol-gel has a concentration of $19-10 \mathrm{mg} / \mathrm{ml}$ and is stable at RT for several months.

\section{Device Fabrication}

The FTO substrates were bought from Solems, model ASAHI 100 by CVD of $1.1 \mathrm{~mm}$ thickness (70-100 ohm resistance, thickness of FTO layer $800 \AA$.) and were etched with a mixture of Zn powder ( $\geq 99 \%$, Aldrich), and hydrochloric acid $(\mathrm{HCl})$ (ACS reagent 37\%from Sigma Aldrich). The etched substrates were then cleaned with soap water, mili-Q water and ethanol (99.5\%), dry with $\mathrm{N}_{2}$ and treated under UV-ozone treatment for 20 min before use.

Once cleaned, a $80 \square 1$ of PZT solution was spin-coated on top of the FTO. The solution was dried in the hot plate for $5 \mathrm{~min}$ at $150^{\circ} \mathrm{C}$. The PZT layer was further annealed $\left(400-650^{\circ} \mathrm{C}\right)$ for $10 \mathrm{~min}$ in air. Depending on the final desired thickness this coating procedure can be repeated up to three times or the PZT solution diluted in acetic acid. The P3HT:PCBM solution (30 mg/ml in Chlorobenzene) was spin-coated on top of the oxide 
layer at $1000 \mathrm{rpm}$, and then, a solution of $\mathrm{V}_{2} \mathrm{O}_{5}$ sol-gel:Isopropanol (1:1) was spin-coated at $1000 \mathrm{rpm}$ and annealed at $120^{\circ} \mathrm{C}$ for $5-10 \mathrm{~min}$ in a hot plate under ambient conditions. Finally a layer of $100 \mathrm{~nm}$ of the $\mathrm{Ag}$ metal was evaporated on top of the $\mathrm{V}_{2} \mathrm{O}_{5}$ layer in a BOC EDWARDS AUTO 306 thermal evaporator using a shadow masks. After device fabrication, thermal annealing was carried out at $120{ }^{\circ} \mathrm{C}$ for 10 minutes in air.

\section{Material and Device Characterization}

$X$-ray powder diffraction (XRD) analyses between 5 and 120 degrees in a RIGAKU Rotaflex RU200 B instrument, using $\mathrm{CuK} \alpha 1$ radiation.

The $X$-ray photoelectron spectroscopy (XPS) and ultraviolet photoelectron spectroscopy (UPS) measurements were performed with a Phoibos 150 analyzer (SPECS GmbH, Berlin, Germany)) in ultra-high vacuum conditions (base pressure 3E-10mbar). XPS measurements were performed with a monochromatic Kalpha xray source $(1486.74 \mathrm{eV})$ and UPS measurements were realized with monochromatic HeI UV source $(21,2 \mathrm{eV})$. Work function determination was done by applying bias of $-10 \mathrm{~V}$ at the sample.

The solar simulation was carried out with a Steuernagel Solarkonstant KHS1200. Light Intensity was adjusted at $1000 \mathrm{~W} / \mathrm{m}$ with a bolometric Zipp\&Konen CM-4 pyranometer. Calibration of the sun simulator was made by several means, with a calibrated S1227-1010BQ photodiode from Hamamatsu and a minispectrophotometer from Ava-Spec 4200. The AM1.5G reference spectrum was according to an ASTMG173 standard. Solar decay and IV-curves were measured using Keithley 2601 multimeter connected to a computer and software.

The UV-visible analysis of solutions and thin films were made in a Shimadzu 1800 spectrophotometer.

The IPCE analyses were carried out with a QE/IPCE measurement System from Oriel at $10 \mathrm{~nm}$ intervals between 300 and $700 \mathrm{~nm}$. The calibration was carried out using a Hamamatsu S1227-101030 diode. The results were not corrected for intensity losses due to light absorption and reflection by the glass support. 
The ferroelectric characterization was performed with a precision LC tester LCII Ferroelectric Test System unit of Radiant technologies attached to a probe station.

The TEM lamella was defined by a focused ion beam. The FIB has circuit editing capability and nanomachining capabilities. The FIB used in this work is a Zeiss 1560XB Cross Beam able to work with sample of size up to a $150 \mathrm{~mm}$. The field emission focused ion beam optics have a liquid Gallium ion emitter with acceleration voltage of $3 \mathrm{kV}$ to $30 \mathrm{kV}$ and beam current of $1 \mathrm{pA}-20 \mathrm{nA}$ with a maximum resolution of $7.0 \mathrm{~nm}$. The system contains a multiple injector needle system for 5 different gases including gas chemistry solution for ion or electron beam deposition of Platinum-containing material.

The TEM inspection has been carried out by a high-resolution FEI Tecnai F20 S/TEM equipped with an EDX. 


\section{Acknowledgements}

APT acknowledges Agencia Estatal de Investigación (AEI) and Fondo Europeo de Desarrollo Regional under contract ENE2015-74275-JIN. All authors acknowledge Dr G. Sauthier for the XPS/UPS measurements, S. Amores for the lamella fabrication and Dr B. Ballesteros for the TEM inspection. The ICN2 is funded by the CERCA programme / Generalitat de Catalunya and by the Severo Ochoa programme of the Spanish Ministry of Economy, Industry and Competitiveness (MINECO, grant no. SEV-2013-0295). To the Spanish MINECO for the grant ENE2016-79282-C5-2-R and the OrgEnergy Excelence Network CTQ2016-81911-REDT.To the Agència de Gestió d'Ajuts Universitaris i de Recerca for the support to consolidated Catalonia research groups 2014SGR 1212 and 1216-F. To the COST Action StableNextSol project MP1307; and to the European Research Council (ERC) Starting Grant, project No. 308023.

\section{Author Contributions}

APT, MLC and GC conceived the experiments. APT was in charge of the experimental and characterization work assisted by AL, QB and IS and. All authors analysed the data and co-wrote the paper. 


\section{References}

[1] J. E. Lilienfeld, patents US1745175A (1925) and US1900018 (1928); J. Bardeen, W. H. Brattain patent US2524035A (1948) and W. Shockley patent US2569347A (1948).

[2] Ferain, I., Colinge, C. A. \& Colinge J.-P. Multigate transistors as the future of classical metal-oxidesemiconductor field-effect transistors. Nature 479, 310-316 (2011).

[3] del Alamo, J. A. Nanometre-scale electronics with III-V compound semiconductors. Nature 479, 317$323(2011)$.

[4] Yi, H., Choi, T., Choi, S., Oh, Y. \& Cheong, S. W. Mechanism of the switchable photovoltaic effect in ferroelectric $\mathrm{BiFeO}_{3}$. Adv. Mater. 23, 3403-3407 (2011).

[5] Ji, W., Yao, K. \& Liang, Y. C. Bulk Photovoltaic Effect at Visible Wavelength in Epitaxial Ferroelectric $\mathrm{BiFeO}_{3}$ Thin Films. Adv. Mater. 22, 1763-1766 (2010).

[6] Zhang, L., Chen, J., Cao, J., He, D. \& Xing, X. Large resistive switching and switchable photovoltaic response in ferroelectric doped $\mathrm{BiFeO}_{3}$-based thin films by chemical solution deposition. J. Mater. Chem. C 3, 4706 (2015).

[7] Yuan, Y., Chae, J., Shao, Y., Wang, Q., Xiao, Z., Centrone, A. \& Huang, J. Photovoltaic Switching Mechanism in Lateral Structure Hybrid Perovskite Solar Cells. Adv. Energy Mater. 5, 1500615 (2015).

[8] Xiao, Z., Yuan, Y., Shao, Y., Wang, Q., Dong, Q., Bi, C., Sharma, P., Gruverman, A. \& Huang, J. Giant switchable photovoltaic effect in organometal trihalide perovskite devices. Nat. Mater. 14, 193-198, (2015).

[9] Nau, S., Wolf, C., Sax, S. \& List-Kratochvil, E. J. W. Organic Non-Volatile Resistive Photo-Switches for Flexible Image Detector Arrays. Adv. Mater. 27, 1048-1052 (2015).

[10] El Gemayel, M., Börjesson, K., Herder, M., Duong, D. T., Hutchison, J. A., Ruzié, C., Schweicher, G., Salleo, A., Geerts, Y., Hecht, S., Orgiu, E. \& Samorì, P. Optically switchable transistors by simple incorporation of photochromic systems into small-molecule semiconducting matrices. Nat. Commun. 6 , $6330(2015)$. 
[11] Kufer, D. \& Konstantatos, G. Photo-FETs: Phototransistors Enabled by 2D and 0D Nanomaterials. ACS Photonics 3, 2197-2210 (2016).

[12] García de Arquer, F. P., Armin, A., Meredith P. \& Sargent, E. H. Solution-processed semiconductors for next-generation photodetectors. Nat. Rev. Mat. 2, 16100 (2017).

[13] Wang, H. \& Kim, D. H. Perovskite-based photodetectors: materials and devices. Chem. Soc. Rev. 46, 5204 (2017).

[14] Börjesson, K., Herder, M., Grubert, L., Duong, D. T., Salleo, A., Hecht, S., Orgiu, E. \& Samorì, P. Optically switchable transistors comprising a hybrid photochromic molecule/n-type organic active layer. J. Mater. Chem. C 3, 4156-4161 (2015).

[15] Strukov, D. B., Snider, Stewart, G. S. \& Williams, R. S. The missing memristor found. Nature 453, 80-83 (2008).

[16] Sze, S. M. \& Ng, K. K. Physics of semiconductor devices. (John Wiley \& Sons, Inc., Hoboken, New Jersey, USA, 2006).

[17] Wakayama, Y., Hayakawa, R. \& Seo, H-S. Recent progress in photoactive organic field-effect transistors. Sci. Technol. Adv. Mater. 15, 024202 (2014).

[18] Pérez-Tomás, A., Lira-Cantú, M. \& Catalan, G. Above-Bandgap Photovoltages in Antiferroelectrics. Adv. Mater. 28, 9644 (2016).

[19] Sturman, P. J. \& Fridkin, V. M. Photovoltaic and Photo-refractive Effects in Noncentrosymmetric Materials (Gordon and Breach Science Publishers, Philadelphia, USA, 1992)

[20] Pérez-Tomás, A., Mingorance, A., Tanenbaum, D. \& Lira-Cantú, M. Metal Oxides in Photovoltaics: AllOxide, Ferroic, and Perovskite Solar Cells (Ch. 8) in The Future of Semiconductor Oxides in NextGeneration Solar Cells. Elsevier, Amsterdam, Netherlands, 267-356, (2018). https://doi.org/10.1016/B978-0-12-811165-9.09989-0

[21] Cao, D., Wang, C., Zheng, F., Dong, W., Fang \& L., Shen, M. High-Efficiency Ferroelectric-Film Solar Cells with an $n$-type $\mathrm{Cu}_{2} \mathrm{O}$ Cathode Buffer Layer. NanoLett. 12, 2803-2809 (2012). 
[22] Zheng, F., Xin, Y., Huang, W., Zhang, J., Wang, X., Shen, M., Dong, W., Fang, L., Bai, Y., Shen \& X., Hao, J. Above $1 \%$ efficiency of a ferroelectric solar cell based on the $\mathrm{Pb}(\mathrm{Zr}, \mathrm{Ti}) \mathrm{O}_{3}$ film. J. Mater. Chem. A, 2, 1363-1368 (2014).

[23] Fan, Z., Yao, K. \& Wang J. Photovoltaic effect in an indium-tin-oxide/ZnO/ $\mathrm{BiFeO}_{3} / \mathrm{Pt}$ heterostructure. Appl. Phys. Lett., 105, 162903 (2014).

[24] Wu, F., Guo, Y., Zhang, Y., Duan, H., Li, H. \& Liu, H. Enhanced Photovoltaic Performance in Polycrystalline $\mathrm{BiFeO}_{3}$ Thin Film/ZnO Nanorod Heterojunctions. J. Phys. Chem. C 118, 15200-15206 (2014).

[25] Loh, L., Briscoe, J. \& Dunn, S. Enhanced performance with bismuth ferrite perovskite in ZnO nanorod solid state solar cells. Nanoscale 6, 7072-7078 (2014).

[26] Tiwari, D., Fermin, D. J., Chaudhuri \& T. K., Ray, A. J. Solution processed bismuth ferrite thin films for all-oxide solar photovoltaics. Phys. Chem. C 119, 5872-5877 (2015).

[27] Zhao, P., Bian, L., Wang, L., Xu, J. \& Chang, A. Photovoltaic effect in an indium-tinoxide/ZnO/BiFeO $3 / \mathrm{Pt}$ heterostructure. Appl. Phys. Lett. 105, 013901 (2014).

[28] Stengel, M., Aguado-Puente, P., Spaldin, N. A. \& Junquera, J. Band alignment at metal/ferroelectric interfaces: Insights and artifacts from first principles. Phys. Rev. B 83, 235112 (2011).

[29] Chen, F. \& Klein, A. Polarization dependence of Schottky barrier heights at interfaces of ferroelectrics determined by photoelectron spectroscopy. Phys. Rev. B 86, 094105 (2012).

[30] Rault, J. E., Agnus, G., Maroutian, T., Pillard, V., Lecoeur, Ph., Niu, G., Vilquin, B., Silly, M. G., Bendounan, A., Sirotti, F. \& Barrett, N. Interface electronic structure in a metal/ferroelectric heterostructure under applied bias. Phys. Rev. B, 87, 155146 (2013).

[31] Yuan, Y., Reece, T. J., Sharma, P., Poddar, S., Ducharme, S., Gruverman, A., Yang Y. \& Huang, J. Efficiency enhancement in organic solar cells with ferroelectric polymers. Nat. Mater. 10, 296-302 (2011).

[32] Yuan, Y., Sharma, P., Xiao, Z., Poddar, S., Gruverman, A., Ducharme, S. \& Huang, J. Understanding the effect of ferroelectric polarization on power conversion efficiency of organic photovoltaic devices. Energy Environ. Sci. 5, 8558-8563 (2012). 
[33] Nalwa, K. S., Carr, J. A., Mahadevapuram, R. C., Kodali, H. K., Bose, S., Chen, Y., Petrich, J. W., Ganapathysubramanian, B. \& Chaudhary, S. Enhanced charge separation in organic photovoltaic films doped with ferroelectric dipoles. Energy Environ. Sci. 5, 7042-7049 (2012).

[34] Shin, K., Kim, T. Y., Yoon, G. C., Gupta, M. K., Kim, S. K., Seung, W., Kim, H., Kim, S., Kim, S. M. \& Kim, S-W. Ferroelectric Coupling Effect on the Energy-Band Structure of Hybrid Heterojunctions with Self-Organized P(VDF-TrFE) Nanomatrices. Adv. Mater. 26, 5619-5625 (2014).

[35] O. Ostroverkhova. Organic Optoelectronic Materials: Mechanisms and Applications. Chem. Rev. 116, 13279-13412 (2016).

[36] Terán-Escobar, G., Pampel, J., Caicedo J. M. \& Lira-Cantú, M. Low-temperature, solution-processed, layered $\mathrm{V}_{2} \mathrm{O}_{5}$ hydrate as the hole-transport layer for stable organic solar cells. Energy Environ. Sci. 6, 3088-3098 (2013).

[37] Nechache, R., Harnagea, C., Li, S., Cardenas, L., Huang, W., Chakrabartty, J. \& Rosei, F. Bandgap tuning of multiferroic oxide solar cells. Nat. Photonics 9, 61 (2014).

[38] Zheng, F., Zhang, P., Wang, X., Huang, W., Zhang, J., Shen, M., Dong, W., Fang, L., Bai, Y., Shen, X., Sun, H. \& Hao, J. Photovoltaic enhancement due to surface-plasmon assisted visible-light absorption at the inartificial surface of lead zirconate-titanate film. Nanoscale 6, 2915 (2014).

[39] Chen, B., Zuo, Z., Liu, Y., Zhan Q.-F., Xie, Y., Yang, H., Dai, G., Li, Z., Xu, G. \& Li, R.-W. Tunable photovoltaic effects in transparent $\mathrm{Pb}\left(\mathrm{Zr}_{0.53}, \mathrm{Ti}_{0.47}\right) \mathrm{O}_{3}$ capacitors. Appl. Phys. Lett. 100, 173903 (2012).

[40] Horiuchi, S. \& Tokura, Y. Organic ferroelectrics. Nat. Mater. 7, 357-366 (2008).

[41] Asadi, K., Li, M., Blom, P. W. M., Kemerink, M \& de Leeuw, D. M. Organic ferroelectric optoelectronic memories. Materials Today 14, 592 (2011).

[42] Shvydka, D. \& Karpov, V. G. Nanodipole photovoltaics. Appl. Phys. Lett. 92, 053507 (2008).

[43] Lazarev, V. V., Blinov, L. M., Yudin S. G. \& Palto, S. P. Photovoltaic effect in an organic semiconductor controlled by a polymer ferroelectric. Crystallography Reports 60, 286-288 (2015).

[44] Hu, L., Dalgleish, S., Matsushita, M. M., Yoshikawa, H. \& Awaga, K. Storage of an electric field for photocurrent generation in ferroelectric-functionalized organic devices. Nat. Commun. 5, 3279 (2014). 
[45] Asadi, K., de Bruyn, P., Blom, P. W. M. \& de Leeuw, D. M. Origin of the efficiency enhancement in ferroelectric functionalized organic solar cells. Appl. Phys. Lett. 98, 183301 (2011).

[46] Wong, F., Perez, G., Bonilla, M., Colon-Santana, J. A., Zhang, X., Sharma, P., Gruverman, A., Dowben, P. A. \& Rosa, L. G. Changing molecular band offsets in polymer blends of (P3HT/P(VDF-TrFE)) poly(3-hexylthiophene) and poly(vinylidene fluoride with trifluoroethylene) due to ferroelectric poling. RSC Adv. 4, 3020-3027 (2014).

[47] Cai, R., Kassa, H. G., Haouari, R., Marrani, A., Geerts, Y. H., Ruzié, C., van Breemen, A. J. J. M., Gelinck, G. H., Nysten, B., Huf, Z. \& Jonas, A. M. Organic ferroelectric/semiconducting nanowire hybrid layer for memory storage. Nanoscale 8, 5968-5976 (2016).

[48] Liu, B., Xu, F., Zhang, X., Yan, D. \& Lu, D. Numerical simulations of the role of a ferroelectric polymer interfacial layer in organic solar cells. Phys. Chem. Chem. Phys. 18, 5412-5418 (2016).

[49] Tiwari, D. \& Dunn, S. Photochemistry on a polarisable semi-conductor: what do we understand today? J. Mater. Sci. 44, 5063-5079, (2009). 


\section{Figure Captions}

Fig. 1. (a) Typical device configuration of a conventional (three-terminal) photo-transistor. Here we propose a new two-terminal phototransistor concept (photo-ferroelectric transistor). (b) Schematic view of the FEoxide/organic semiconductor (OS) photovoltaic switch with a configuration TCO/FE-oxide/OS/HTM/Metal. The cathode TCO (transparent conductive oxide) is fluorine doped tin oxide (FTO). The ferroelectric layer is a compact PZT thin-film with formula $\mathrm{Pb}\left(\mathrm{Zr}_{x} \mathrm{Ti}_{1-x}\right) \mathrm{O}_{3}(x=0.53)$. The organic semiconductor (OS) absorber is a bulk heterojunction thin film made of the P3HT polymer, donor, and the PCBM fullerene, acceptor and the hole transport material (HTM) is a layer of $\mathrm{V}_{2} \mathrm{O}_{5}$. The metal anode is silver. (c) Experimental energy band alignment of the (unpoled) photovoltaic transistor structure as determined from UPS, XPS and UV-Vis. Electrons and holes are photo-generated in the OS absorbing layer and selectively collected by the ETM and HTM and transported to the outer FTO and Ag electrodes, respectively. Poling of the structure results in a modification of the band alignment of the PZT/PCBM interface and a transistor effect.

Fig. 2. (a) Representative hysteresis loop of the FTO/PZT/Ag capacitor. The optimization of the FEoxide/P3HT:PCBM short-circuit current was carried out by means of the PZT annealing temperature (i.e. ferroelectric polarization) and the PZT concertation (i.e. PZT thickness). In this panel, (b) represents the FEoxide/P3HT:PCBM short-circuit current as a function of the PZT anneal temperature. A total of 40 devices were fabricated for each temperature in 5 different batches. (c) Illustrates the evolution of $j_{s c}$ as a function of the concentration of the $0.4 \mathrm{M} \mathrm{Pb}-\mathrm{Zr}-\mathrm{Ti}$ solution in parts of acetic acid $(c / c 0)$. A total of 74 devices were fabricated in 7 different batches (4-5 for each c/c0).

Fig. 3. Poling the device as depicted in (a) and (b) leads to significant variations of the FE-oxide/PCBM LUMO band offset ( $\square E_{c}$ ). Downward polarization (c) facilitates current flow while upward polarization (d) enhances the FE-oxide/OS $n$-type LUMO band offset. The conduction band offset acts as an electron barrier blocking the photovoltaic current (off-state of the ferroelectric photovoltaic transistor). 
Fig. 4. (a) Photovoltaic current AM1.5G $\left(1000 \mathrm{~W} / \mathrm{m}^{2}\right)$ of the FE-oxide/P3HT:PCBM photovoltaic transistor showing the on and off states (both under illumination). (b) Dark current on and off state showing already resistive switching behavior. (c) The switchable behavior is consistent after subsequent poling cycles. 
Figures
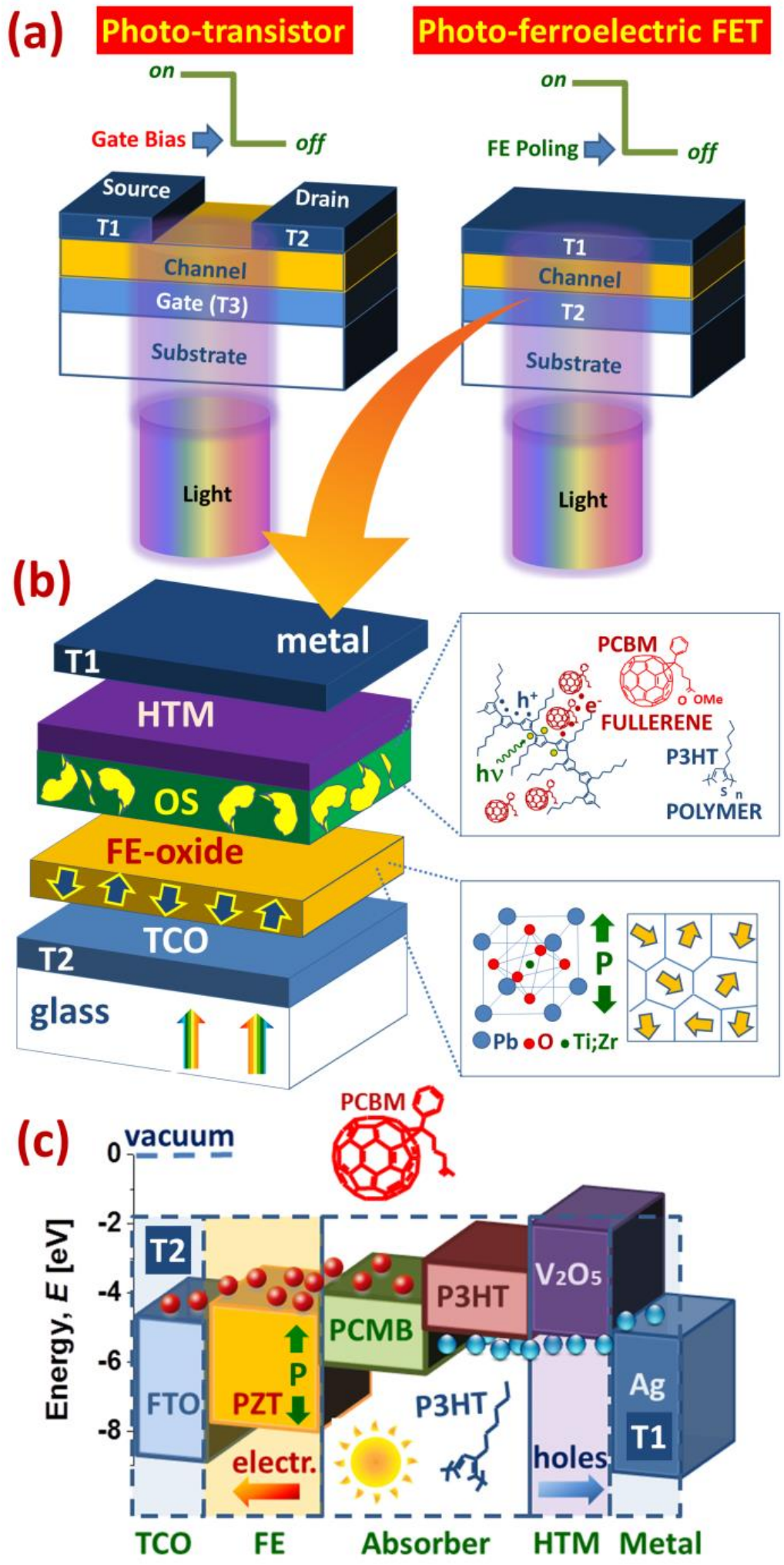

Fig. 1. 


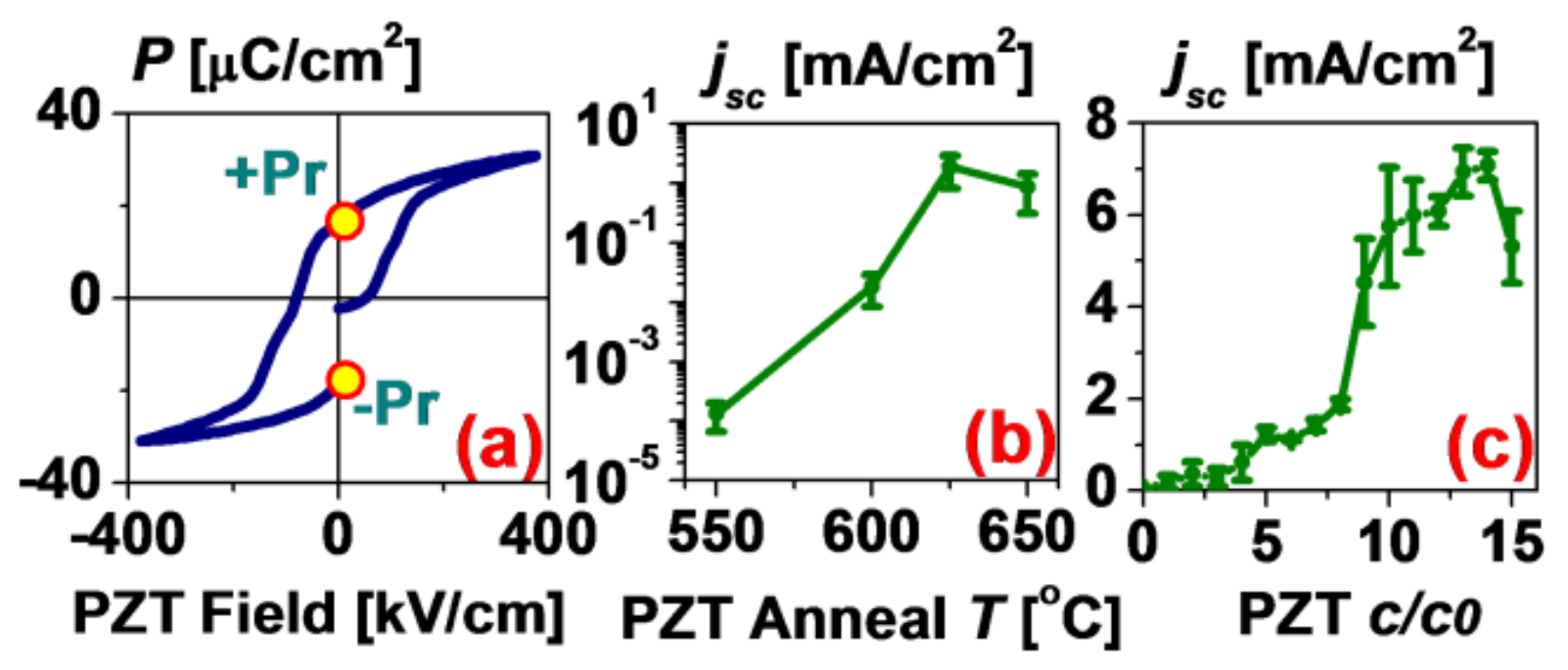

Fig. 2. 

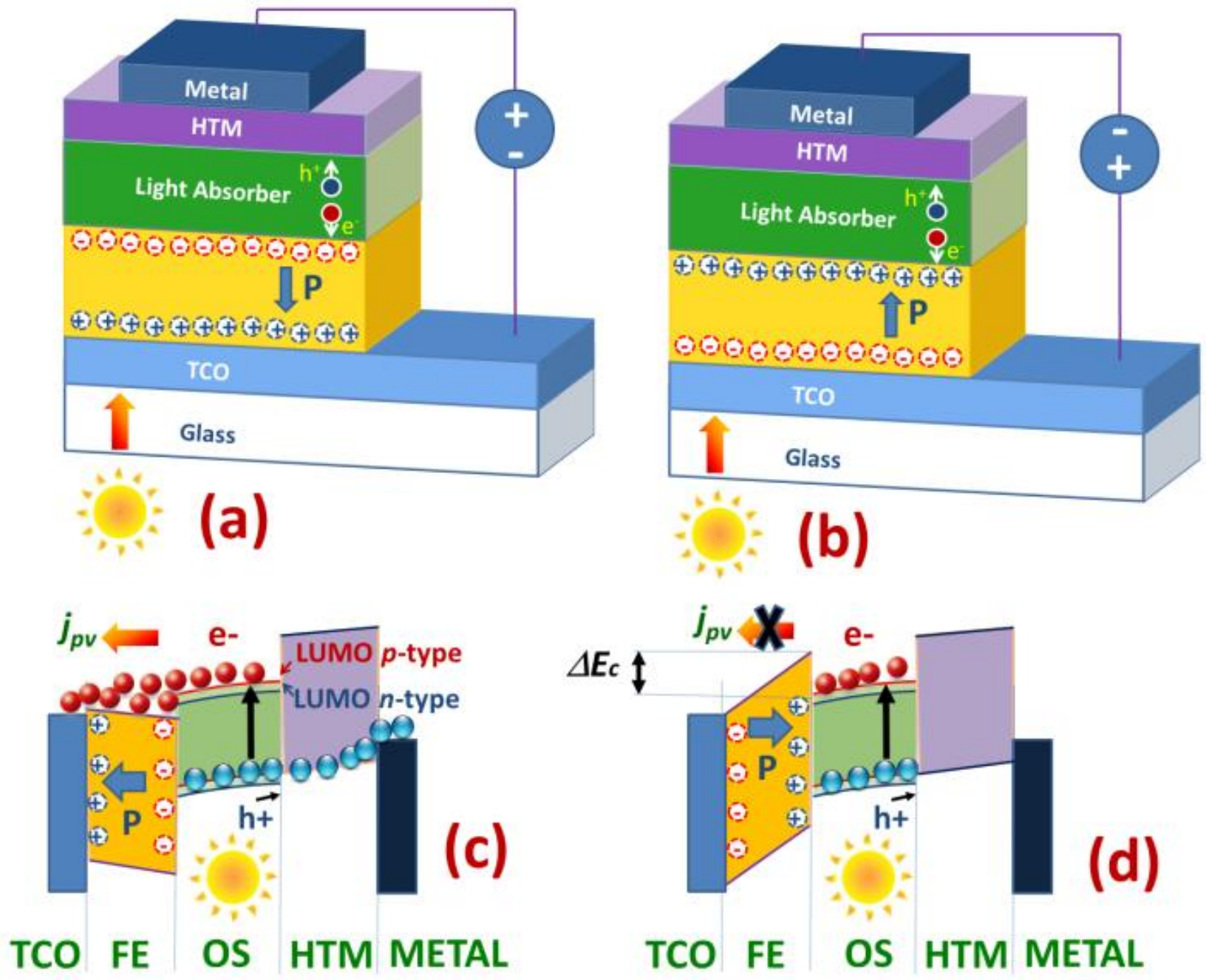

on-state

off-state

Fig. 3. 

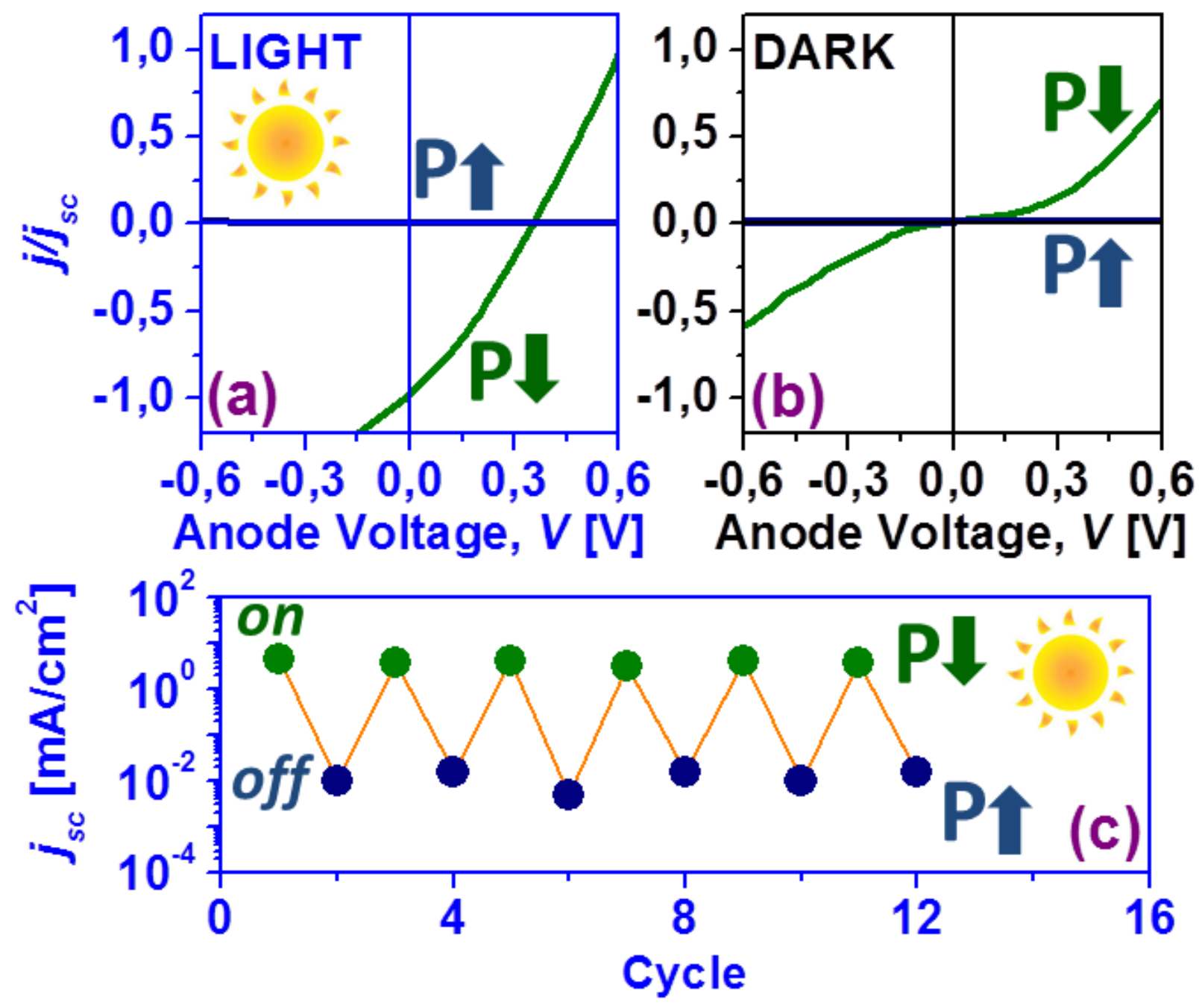

Fig. 4. 